\title{
Myasthenic crisis: Clinical features, complications and mortality
}

\author{
J. M. K. Murthy, A. K. Meena*, G. V. S. Chowdary, Jaishree T. Naryanan \\ Department of Neurology, The Institute of Neurological Sciences, Care Hospital and *Nizam's Institute of Medical Sciences, Hyderabad, India
}

\begin{abstract}
Background and Objective: Myasthenic crisis is a lifethreatening complication of myasthenia gravis (MG) and when treated aggressively is associated with good outcome. Materials and Methods: Retrospective study of case records of patients with episodes of myasthenic crisis. Results: Twenty-one (22\%) of the 95 patients with MG (9 with thymoma), experienced 23 episodes of myasthenic crisis, $3(33 \%)$ in patients with thymoma. The crisis episodes occurred within 2 years of disease onset in $11(52 \%)$ patients. Infection was the most common primary precipitant of the crisis occurring in $65 \%$. The median duration of the crisis episode was 11 days (7-39 days), and the median neurological intensive care unit stay was 15 days (range 947 days). Fifteen $(65 \%)$ episodes were treated with small volume plasma exchange (PE) and 8 (35\%) episodes received intravenous immunoglobulin (IVIg). The time taken for disease stabilization, the median number of days for extubation, was 8 days (range 7-12) in the PE group and 10 days (range 7-39) in the IVIg group. Disease stabilization could not be achieved in one patient in the IVIg group. Ventilator-associated pneumonia (VAP) was the commonest complication, seen in $30 \%$. Two (8\%) of the 23 episodes of crisis were fatal, one resulting from VAP and septicemia, and the other due to crisis itself. All the 19 patients who survived to discharge had complete resolution of admission symptoms. Conclusions: In patients with myasthenic crisis, both therapeutic options, PE and IVIg, are equally effective in disease stabilization. To achieve good outcomes all efforts should be directed at decreasing the duration of intubation and also aggressively treating the associated medical complications.
\end{abstract}

Key Words: Myasthenic crisis, plasma exchange, intravenous immunoglobulins, mechanical ventilation

Myasthenia gravis (MG) an autoimmune disorder of the neuromuscular junction tends to be characterized by exacerbations and remissions. Myasthenic crisis is a serious complication of MG and is defined as weakness from acquired MG that is severe enough to require intubation, ${ }^{[1]}$ a situation that corresponds to Class V of the Myasthenia Gravis Foundation of America (MGFA) classification. ${ }^{[2]}$ MGFA considers the most severely affected muscle to define the patient's Class and avoided defining "myasthenic crisis". Class IV and Class $\mathrm{V}$ of MGFA clinical classification correspond to severe myasthenia. Myasthenic crisis may complicate in 15 to $20 \%$ of patients with MG. ${ }^{[3,4]}$

There is limited data on myasthenic crisis from India. ${ }^{[5]}$ Of the neuromuscular disorders admitted to a tertiary teaching hospital neurological intensive care unit (NICU), myasthenia crisis accounted for $7.5 \%{ }^{[6]}$ The only documented data on this topic, reported intensive care in eight patients with myasthenic crisis. ${ }^{[7]}$

\section{Materials and Methods}

We retrospectively identified all the patients with episodes of myasthenic crisis from the case records of the patients with myasthenia gravis seen by the senior author. Diagnosis of MG was established by neostigmine test and decrement response. Myasthenic crisis was defined as weakness from acquired MG that is severe enough to require intubation. ${ }^{[1]}$ Patients with perioperative respiratory crisis associated with thymectomy were not included.

From the case records demographic data, duration of the disease, clinical features, precipitating factors, duration of mechanical ventilation, complications seen during the crisis period, the treatment received, and the outcome were recorded. Diagnosis of atelectasis and ventilator-associated pneumonia was based on radiological, clinical, and laboratory findings.

Intubation criteria included impaired swallowing mechanism leading to inability to clear secretions, ineffective cough and nasal voice, signs of pneumonia with marginal $\mathrm{PO}_{2}$ or widening $\mathrm{A}$-a gradient, and $\mathrm{VC}$ below $15 \mathrm{~mL} / \mathrm{kg}^{\left[{ }^{[8]}\right.}$ All patients were managed on synchronous intermittent mandatory ventilation (SIMV) with the settings: 
tidal volume of $\sim 12 \mathrm{~mL} / \mathrm{kg}$, respiratory rate of $12 / \mathrm{min}$ and positive end-expiratory pressure (PEEP) 3-5 mm Hg. Weaning criteria included $\mathrm{PaO}_{2}$ greater than $80 \mathrm{mmHg}$ with $\mathrm{FiO}_{2}$ of $40 \%, \mathrm{PCO}_{2}$ less than $42 \mathrm{mmHg}$, and a spontaneous respiratory rate less than 20 . Tpiece trial was used while weaning the patient off the ventilator.

Treatment, plasma exchange (PE) or intravenous immunoglobulin (IVIg), was based on the accessibility to PE services and affordability to the patient. In the PE group, five cycles of small volume exchange were performed on alternate days. Each session consisted of two sittings and for each sitting $8-10 \mathrm{ml} / \mathrm{kg}$ of plasma was exchanged, a maximum of $500 \mathrm{ml}$ for each sitting. Treatment regimen for IVIg consisted of $400 \mathrm{mg} / \mathrm{kg} /$ day administrated for 5 days. The outcome recorded included the number of days taken for disease stabilization. This was measured by the time taken to extubate the patient.

\section{Results}

Of the 95 patients with MG including 9 patients with thymoma seen by the senior author, $21(22 \%)$ patients had 23 episodes of myasthenic crisis. Median age at the onset of crisis was 40 years (range 28-75 yrs) and the ratio of men to women was 1.3:1. The crisis episodes occurred within 2 years (range 9-24 months) of disease onset in $11(52 \%)$ patients. Thymoma was present in $14 \%(3 / 21)$ of patients with crisis, on the other hand $3(33.3 \%)$ of the 9 patients with thymoma developed crisis; one of them had 3 crisis episodes. This patient also had associated cholinergic overstimulation (Table 1).

Weakness involved the respiratory, oropharyngeal, and neck muscles in $95 \%$ of the patients at the time of intubation. All patients had generalized weakness in addition. Infections were the most common primary precipitants of crisis occurring in $65 \%$ of patients and in one patient it was related to poor drug compliance (Table 2).

The median duration of the crisis episode was 11 days (range 7-39 days), and median NICU stay was 15 days (range 9-47 days). One patient who needed assisted ventilation had severe

\begin{tabular}{|c|c|c|c|}
\hline \multicolumn{4}{|c|}{ Table 1: Frequency of myasthenic crisis } \\
\hline & $\begin{array}{l}\text { Early onset } \\
(<40 \text { yrs }) \\
n=45 \\
8(38 \%)\end{array}$ & $\begin{array}{l}\text { Thymoma } \\
\mathbf{n}=\mathbf{9}\end{array}$ & $\begin{array}{l}\text { Late onset } \\
(>40 \text { yrs) } \\
n=41\end{array}$ \\
\hline $\begin{array}{l}\text { Number of Patients } \\
\text { in crisis }(n=21)\end{array}$ & $8(38 \%)$ & & \\
\hline $\begin{array}{l}\text { Within } 2 \text { years of disease } \\
\text { onset }(n=11)\end{array}$ & $3(27 \%)$ & $2(18 \%)$ & $6(55 \%)$ \\
\hline \multicolumn{4}{|c|}{$\begin{array}{l}{ }^{*} \text { One of the patients had } 3 \text { episodes of myasthenic crisis and episodes were } \\
\text { associated with cholinergic over-stimulation; }{ }^{* *} \text { One patient presented with } \\
\text { crisis }\end{array}$} \\
\hline
\end{tabular}

\begin{tabular}{lcc}
\hline \multicolumn{3}{c}{ Table 2: Myasthenic crisis - risk factors } \\
\hline Infections & 10 & $15(65.0)$ \\
$\quad$ Bronchopneumonia & 4 & \\
$\quad$ Viral fever & 1 & \\
UTI & & $1(4.5)$ \\
Drug withdrawal & & $7(30.5)$ \\
Unknown &
\end{tabular}

Parenthesis are percentage depression. Whenever an attempt was made to wean him off the ventilator he used to get panic attacks. During these episodes he used to hyperventilate and get exhausted resulting in low tidal volume. All the patients were managed on SIMV ventilator mode. Cholinergic medication was stopped during the period of mechanical ventilation. Hospital acquired infections were the common complications. Ventilator-associated pneumonia $(30 \%)$ was the commonest infection (Table 3).

Fifteen $(65 \%)$ episodes were treated with small volume PE and $8(35 \%)$ episodes received IVIg. The outcome was similar with both the treatment modalities. In none of the patients were the treatments stopped prematurely. The time taken for the disease to stabilize, the median number of days for extubation, was 8 days (range 7-12) in the PE group and 10 days (range 7-39) in the IVIg group. Disease stabilization could not be achieved in one patient in the IVIg group (Table $4)$.

Two patients in the PE group developed hypotension during one of the exchange schedules. This could be corrected with volume expansion. One patient in the IVIg group developed elevation in blood urea and serum creatinine after four doses of IVIg. However he was given the fifth dose without any adverse events. With conservative treatment urea creatinine levels became normal.

Two $(8 \%)$ of the 23 episodes of crisis were fatal, one resulted from ventilator- associated pneumonia and septicemia, the other was related to crisis. In this patient disease stabilization could not be achieved with IVIg; while she was on T-piece trial, she had sudden cardiac arrest, probably related to cardiac arrhythmia, from which she could not be revived. All the 19 patients who survived to discharge had complete resolution of admission symptoms. All patients were

Table 3: Complications during the period of crisis $(\mathrm{N}=23)$

\begin{tabular}{ll}
\hline Complication & $(\%)$ \\
Ventilator associated pneumonia & $7(30)$ \\
Atelectasis & $4(17)$ \\
UTI & $4(17)$ \\
Sepsis & $2(8)^{\star}$ \\
Diarrhea & $3(13)^{\star *}$ \\
Cholinergic over-stimulation & $1(4)$ \\
Depression & $1(4)$
\end{tabular}

*One of the patients with septicemia was associated with ventilator-associated pneumonia; ${ }^{*}$ In one patient the diarrhea could be attributed to cholinergic over-stimulation

Table 4: Myasthenic crisis - treatment protocols, complications and mortality

Plasma exchange IV immunoglobulins

\begin{tabular}{lcc} 
MG without thymoma & $(\%)$ & $(\%)$ \\
MG with thymoma & $3(20)$ & $6(75)$ \\
Median duration (days) & $8(7-12)$ & $2(25)$ \\
Complications & $2(13)$ & $10(7-39)$ \\
Mortality & $1^{*}(7)$ & $1(12.5)$ \\
\hline
\end{tabular}

*This patient died due to ventilator-associated pneumonia and septicemia; ${ }^{* *} \ln$ this patient disease stabilization could not be achieved and she died probably due to cardiac arrhythmia 
started on steroids before discharge.

\section{Discussion}

Myasthenic crisis frequently occurs within the first 2 years after disease onset, ${ }^{[3]}$ and about one-fifth of the patients develop crisis episodes within the first year. ${ }^{[9]}$ Most often myasthenic crisis develops in patients with generalized myasthenia. Rarely, some patients may experience respiratory failure without generalized weakness. ${ }^{[10]}$ A third of the patients who survive the first crisis experience a second crisis. Myasthenic crisis is more common in patients of myasthenia gravis associated with thymoma. ${ }^{[3,4]} \mathrm{A}$ cholinergic crisis is less common and a combination of both crises is often clinically encountered. ${ }^{[4]}$ All the three episodes of myasthenic crisis in one of the patients with thymoma were associated with cholinergic overstimulation. Infections are the most common precipitating factors, $30-40 \%$ of cases. Several drugs which affect the neuromuscular junction are also associated with myasthenic crisis. ${ }^{[1,4]}$ However; no risk factors are identified in 30 to $40 \%$ of patients with myasthenic crisis. ${ }^{[4]}$

Early intubation and mechanical ventilation is perhaps the most important step in the management of myasthenic crisis. This can be achieved by instituting assist-control (AC) or SIMV. Although large tidal volumes have been recommended, ${ }^{[4]}$ more recent literature defends that smaller tidal volumes (7$8 \mathrm{~mL} / \mathrm{kg}$ ) with faster respiratory rates (12-16 breaths/min) should be used to avoid lung injury adding intermittent sighs (1.5 x tidal volume, 3 to 4 times every hour) to avoid atelectasis. ${ }^{[3]}$ Nomori and Ishihara ${ }^{[11]}$ have managed five patients with MG with pressure-controlled ventilation via a mini-tracheotomy tube (MTT). The MG patients could be converted from conventional volume-controlled ventilation to pressure-controlled ventilation $(\mathrm{PCV})$ via MTT. Clinically, PCV via MTT achieved adequate ventilation without impairing their speech. Non-invasive bi-level positive pressure ventilation (BiPAP) has also been used successfully in patients with respiratory failure caused by myasthenic crisis. This study suggests BiPAP may prevent intubation in patients with myasthenic crisis. Eleven episodes of crisis in 9 patients were initially managed with BiPAP and endotracheal intubation was avoided in 7 of these 11 trials. The presence of hypercapna with $\mathrm{PaCO}_{2}>50$ $\mathrm{mmHg}$ at onset predicted BiPAP failure and subsequent intubation. ${ }^{[12]}$

Most patients require ventilation for a brief period, less than two weeks. ${ }^{[3,13]}$ In the series by Thomas and colleagues, ${ }^{[3]}$ half of the patients were extubated by 13 days, and $75 \%$ by 31 days. In this study a pre-intubation serum bicarbonate level $>30 \mathrm{mg} / \mathrm{dl}$, a post-intubation vital capacity of $<25 \mathrm{ml} / \mathrm{kg}$ on day 3 or later; and age $>50$ years have been shown to be independent predictors of prolonged crisis. These clinical variables may serve as clinically useful criteria for estimating the need for mechanical ventilation beyond two weeks. Atelecta- sis, and ventilator-associated pneumonia are the common complications of prolonged mechanical ventilation. ${ }^{[3,14]}$

Plasma exchange and intravenous immunoglobulin are the two immune-directed treatments used for patients with actual or impending myasthenic crisis. Studies comparing PE with IVIg have demonstrated equal efficacy but significantly fewer and less severe side effects for the IVIg treatment. Improvement usually begins within a few days of treatment. ${ }^{[15,16]}$ Studies of daily PE compared with every other day exchange have demonstrated no statistically significant improvement in the strength or number of days of mechanical ventilation but have shown a trend towards improved strength at the end of therapy without increased side effects. ${ }^{[17]}$ However, in a retrospective study PE (compared with IVIg) was associated with a superior ventilatory status at 2 weeks and one-month functional outcome. However, the complication rate was higher with PE. ${ }^{[18]}$ Our study had also demonstrated equal efficacy of both treatments and in the IVIg group disease stabilization could not be achieved in one patient who died. We gave small-volume PE. For patients with severe crisis, more than one course of PE or IVIg may be necessary. A retrospective analysis showed the failure rate of one course of IVIg treatment to be $20 \%{ }^{[18]}$

Advancement in the critical care techniques and the introduction of immunomodulation therapy have dramatically reduced the mortality rates of myasthenic crisis. A recent study suggests that aggressive respiratory treatment (use of suction, intermittent positive pressure breathing or bronchodilator treatments, sighs, and chest physiotherapy) can lower the risk for atelectasis and ventilotor-associated pneuomonia. ${ }^{14]}$ Before 1955, myasthenic crisis was fatal in $80 \%$ of episodes. ${ }^{[19,20]}$ In the recent Columbia series the mortality rate was $10 \%$ overall, half of those dying after extubation. ${ }^{[3]}$ In the Columbia series all deaths were attributable to severe medical comorbidity. The mortality in our series was $9.5 \%$ and one of the deaths was probably related to cardiac arrhythmias. Cardiac arrhythmias occur in up to $17 \%$ of patients with myasthenic crisis. ${ }^{[21-23]}$

Certain limitations of our study must be acknowledged. This was a retrospective study and had a limitation of available clinical data. The treatment allocation between PE and IVIg was not randomized and was based on the accessibility to PE services and affordability to the patients. In spite of such limitations this series suggests that both PE and IVIg are equally effective and safe. To achieve good outcomes all efforts should be directed at decreasing the duration of intubation and also aggressively treating the associated medical comorbidity and complications.

\section{References}

1. Kirmani JF, Yahia AM, Qureshi AI. Myasthenic crisis. Curr Treat Options Neurology 2004;6:3-15.

2. Jaretzki A III, Baroho RJ, Ernstoff RM, Kaminski HJ, Keesey JC, Penn AS, 
et al. Myasthenia gravis recommendations for clinical research standards: Task Force of the Medical Scientific Advisory Board of the Myasthenia Gravis Foundation of America. Neurology 2000;55:16-23.

3. Thomas CE, Mayer SA, Gungor Y, Swarup R, Webster EA, Chang I, et al. Myasthenic crisis: Clinical features, mortality complications and risk factors for prolonged intubation. Neurology 1997;48:1253-60.

4. Mayer SA. Intensive Care of the myasthenic patient. Neurology $1997 ; 48:$ S $70-\mathrm{S} 75$.

5. Panda S, Goyal V, Behari M, Singh S, Srivastava T. Myasthenic crisis: A retrospective study. Neurol India 2004;52:453-6.

6. Meena AK, Prasad VS, Murthy JMK. Neurological intensive care in India Disease spectrum and outcome. Neurol India 2001;49:S1-S7.

7. Aggarwal AN, Gupta D, Behara D, Prabhakar S, Jindal SK. Intensive respiratory care in patients with myasthenic crisis. Neurol India 2001;50:348-51.

8. Wijdick EFM. The clinical practice of critical care neurology. Oxford, University Press, $2^{\text {nd }}$ Ed. 2003. p. 422-36.

9. Juel VC. Myasthenia gravis: Management of myasthenic crisis and perioperative care. Semin Neurol 2004;24:75-81.

10. Putman MR, Wise RA. Myasthenia gravis and upper airway obstruction. Chest 1996:109:400-04

11. Nomori H, Ishihara T. Pressure-controlled ventilation via a mini-tracheostomy tube for patients with neuromuscular disease. Neurology 2000;55:698-702.

12. Rabistein A, Wijdicks EFM. BiPAP in acute respiratory failure due to myasthenic crisis may prevent intubation. Neurology 2002;59:1647-9.

13. Gracey DR, Divertie MB, Howard FM. Mechanical ventilation for respiratory failure in myasthenia gravis. Two-year experience with 22 patients. Mayo Clin Proc 1983;58:597-602.

14. Varelas PN, Chua HC, Natterman J, Barmadia L, Zimmerman P, Yahia A, et al. Ventilatory care in myasthenia gravis crisis: Assessing the baseline adverse event rate. Crit Care Med 2002;30:2663-8.

15. Gajdos P, Chevert S, Clair B, Tranchant C, Chastang C. Clinical trial of plasma exchange and high-dose intravenous immunoglobulin in myasthenia gravis. Myasthenia Gravis Clinical Study. Ann Neurol 1997;41:789-96.

16. Ronager J, Ravnborg M, Hermansen I, Vorstrup S. Immunoglobulin treatment versus plasma exchange in patients with chronic moderate to severe myasthenia gravis. Artif Organs 2001;25:967-73.

17. Yeh JH, Chiu HC. Plasmapheresis in myasthenia gravis: A comparative study of daily versus alternately daily schedule. Acta Neruol Scand 1999;99:147-51.

18. Qureshi AI, Choudary MA, Akbar MS, Mohammad Y, Chua HC, Yahia AM, et al. Plasma exchange versus intravenous immunoglobulin treatment in myasthenic crisis. Neurology 1999;52:629-32.

19. Tether JE. Management of myasthenic and cholinergic crises. Am J Med $1955 ; 19: 740-2$.

20. Osserman KE, Genkins G. Studies in myasthenia gravis: Reduction in mortality rate after crisis. JAMA 1963;183:97-101.

21. Bedlack RS, Sandrs DB. How to handle myasthenic crisis. Postgrad Med $2000 ; 107: 211-4 ; 220-2$.

22. Klein CM, Vernino S, Lennon VA, Sandroni P, Fealey RD, Benrud-Larson L, et al. The spectrum of autoimmune autonomic neuropathies. Ann Neurol 2003;53:752-3

23. Vernino S, Low PA, Fealey RD, Stewart JD, Farrugia G, Lennon VA. Autoantibodies to ganglionic acetylcholine receptors in autoimmune autonomic neuropathies. N Eng J Med 2000;343:847-55.

Accepted on 23.12.2004.

\section{Invited Comments}

One of the two major neuromuscular diseases that neurointensivists admit to an intensive care unit (ICU) is myasthenia gravis (MG, the other, Guillain-Barre syndrome). Because MG patients develop crisis leading to intubation and mechanical ventilation infrequently, not much is known about it. Even less is known about myasthenic crisis in developing countries. The reason is the rarity of the disease per se. Modern studies from Columbia-Presbyterian and Johns Hopkins Hospitals have been published in the Western literature, but even these report few tens of cases. From these and other studies it became evident that in recent times the prognosis of myasthenic crisis has improved significantly. This is mainly due to novel treatments (such as plasmapheresis and IVIG), but, probably to a greater degree, due to early recognition and aggressive treatment of respiratory or infectious complications (such as atelectasis and aspiration pneumonia) in the ICU. For example, a more aggressive respiratory management protocol at Hopkins halved the prevalence of atelectasis and pneumonia compared to the Columbia series. Thus, any study reporting the management or outcomes of this rare situation is welcomed.

From that perspective, the report by Murthy et al, in this issue of Neurology India represents a valuable new insight into the vast Indian population. The authors retrospectively studied 21 MG patients with 23 myasthenic crisis episodes. More than half of these occurred within the first 2 years from the disease onset and in two-thirds the precipitant factor was infectious. Half of the crises lasted for 11 days, with an NICU stay of 15 days, numbers not different from those reported from Western ICUs. Interestingly, although selection bias is quite possible, it seems that plasmapheresis leads to earlier extubation than IVIG. All this information and the fact that only two patients (10\%) expired, shows again the significant improvement in MG crisis outcomes, which is not limited to Western countries anymore. In conclusion, not only for presenting this new data to us, but for their everyday effort and hard clinical work leading to these results, the authors have to be commended.

Panayiotis N. Varelas

Department of Neurology, Director NICU Henry Ford Hospital, 2799 West Grand Boulevard, Detroit, MI 48202-2689, USA.

E-mail: varelas@neuro.hfh.edu 